\title{
Nomenclatural notes and new combinations on Anathallis and Specklinia (Orchidaceae)
}

\author{
Notas nomenclaturais e novas combinações em Anathallis e Specklinia (Orchidaceae)
}

\author{
Fábio de Barros ${ }^{1,3}$ \& Felipe Fajardo V.A. Barberena ${ }^{2}$
}

\begin{abstract}
Even though many new combinations have been proposed for the genera Anathallis Barb.Rodr. and Specklinia Lindl., since their re-establishment in 2001, several Brazilian species bearing the characteristics of these genera were never transferred from Pleurothallis R.Br. sensu lato. In this work 17 Brazilian species formerly attributed to Pleurothallis are transferred to Anathallis (16 species) and Specklinia (one species). Also, several superfluous names created from double new combinations in the genera Anathallis and Specklinia are pointed out.

Key words: nomenclature, Pleurothallidinae, Pleurothallis, taxonomy.

\section{Resumo}

Mesmo após o grande número de novas combinações propostas para os gêneros Anathallis Barb. Rodr. e Specklinia Lindl., desde que foram restabelecidos em 2001, algumas espécies brasileiras que possuem as características desses gêneros nunca foram transferidas do gênero Pleurothallis R.Br. sensu lato. Neste trabalho 17 espécies brasileiras, antes atribuídas a Pleurothallis, são transferidas para Anathallis (16 espécies) e Specklinia (uma espécie). Além disso, alguns nomes supérfluos, decorrentes de novas combinações propostas em duplicatas nos gêneros Anathallis e Specklinia, são apontados.

Palavras-chave: nomenclatura, Pleurothallidinae, Pleurothallis, taxonomia.
\end{abstract}

\section{Introduction}

Since the re-establishment of Anathallis Barb.Rodr. by Pridgeon \& Chase (2001), when the authors attributed 90 binomials to the genus, many new transfers have been proposed by other authors (Azevedo \& Van den Berg 2005; Barros 2002, 2003, 2004, 2006; Barros \& Pinheiro 2002; Luer 2004, 2007, 2008). Today, Anathallis, including Panmorphia Luer (Luer 2006), comprises about 115 binomials, but some species are still in need of transfer, most of them described originally as members of the collective genus Pleurothallis R.Br. sensu lato. During the preparation of the list of orchids for the Catalogue of Brazilian Plants and some other floristic surveys, the authors have found several species of Pleurothallis which agree better with the circumscription of Anathallis, but have never been transferred.

In the same way, Specklinia Lindl. was reestablished by Pridgeon \& Chase (2001) with 86 species, and new transfers were proposed later by Barros $(2004,2005,2006)$ and Luer $(2004,2007)$. Today there are, about 420 binomials attributed to the genus but the actual number of species in Specklinia is hard to estimate, since there are different interpretations on its composition. For instance, Luer (2004) transferred 361 species to Specklinia, most of them treated under other genera like Arthrosia Luer and Panmorphia Luer in his later work on systematics of Specklinia and vegetatively similar taxa (Luer 2006). A partial revision of Speckliniawas presented by Luer (2006) which deals only with 39 species, excluding those endemic to Brazil.

In this work the authors transfer 16 species to Anathallis and one species to Specklinia, and also elucidate the correct nomenclatural status of some species previously transferred to this genus.

\section{Material and Methods}

The folowing herbaria were considered during the elaboration of this work (acronyms according to Holmgren et al. 1990): HB, HUEFS, RB, SP and SPF, as well as the photos of the types available on the sites of the herbaria AMES and NY (respectively 
http://asaweb.huh. harvard.edu:8080/databases/ specimen_index.html, and http://sciweb.nybg.org/ science2/hcol/vasc/ index.asp). The types or photos of the types examined were marked with an exclamation mark.

Lectotypes were proposed for some species when any of the elements of the protologue are available. This was impossible for species known only from the original descriptions and whose holotypes are lost.

Additional material were examined and presented when available.

\section{Results and Discussion}

The following 16 species have the characteristics of the genus Anathallis instead of Pleurothallis sensu stricto, and need to be transferred. They have a ramicaul about as long as the leaves; with an annulus, the lateral sepals in general are free and acute to acuminate, the lip is hinged and bears two little lateral retrorse finger-like expansions at the base, and the column is elongate with a single and ventral stigmatic cavity.

Anathallis bocainensis (Porto \& Brade) F.Barros \& Barberena, comb. nov. Pleurothallis bocainensis Porto \& Brade, Anais Reunião Sul-Amer. Bot. 3: 37. 1938(1940). Type: BRAZIL. SÃO PAULO: Bananal, Serra da Bocaina, V.1936, fl., A.C. Brade 15723 (holotype RB!).

Pleurothallis pfisteri Braem \& Braas, Orchidee (Hamburg) 31: 251, figs. a-j. 1980. Type: BRAZIL. SANTA CATARINA: 1979, G. Pfister s.n. (holotype K).

Anathallis caroli (Schltr.) F.Barros \& Barberena, comb. nov. Pleurothallis caroli Schltr., Repert. Spec. Nov. Regni Veg. Beih. 35: 52. 1925. Type: BRAZIL. RIOGRANDEDOSUL: Rio Pardo, Fazenda Soledade, II.1922, fl., C. Jürgens 64 (holotype B, destroyed).

Up to now this species is known only from the type collection which was destroyed during the World War II.

Anathallis fastigiata (Luer \& Toscano) F.Barros \& Barberena, comb. nov. Pleurothallis fastigiata Luer \& Toscano, Selbyana 23: 186. 2002. Specklinia fastigiata (Luer \& Toscano) Luer, Monogr. Syst. Bot. Missouri Bot. Gard. 95: 260. 2004. Panmorphia fastigiata (Luer \& Toscano) Luer, Monogr. Syst. Bot. Missouri Bot. Gard. 105: 177. 2006. Type:
BRAZIL. PARÁ: Castanhal, Rio Apeú, flowered under cultivation, 16.I.1997, A.L.V. Toscano-deBrito 2291 (holotype HUEFS, not found).

Although Luer \& Toscano-de-Brito (2002) pointed out that the holotype was deposited at the herbarium of the State University of Feira de Santana (HUEFS) we are unable to find it there, and according to the staff of this herbarium apparently it has never been deposited there. Despite this fact the description and the illustration show that the species has the characteristics of Anathallis.

Anathallis funerea (Barb.Rodr.) F.Barros \& Barberena, comb. nov. Lepanthes funerea Barb.Rodr., Vellosia, ed. 2, 1: 118. 1891. Pleurothallis funerea (Barb.Rodr.) Cogn. in Martius, Eichler \& Urban, Fl. bras. 3(4): 567. 1896. Specklinia funerea (Barb.Rodr.) Luer, Monogr. Syst. Bot. Missouri Bot. Gard. 95: 260. 2004. Panmorphia funerea (Barb.Rodr.) Luer, Monogr. Syst. Bot. Missouri Bot. Gard. 105: 156. 2006. Type: BRAZIL.AMAZONAS: rio Yauapery, J.B. Rodrigues s.n. (holotype RB, destroyed); lectotype, here designated, fig. D, tab. 200, vol. 3 (J. Barbosa Rodrigues, Iconographie des Orchidées du Brésil, reproduced in Sprunger (1996, p.258)).

Anathallis globifera (Pabst) F.Barros \& Barberena, comb. nov. Pleurothallis globifera Pabst, Arch. Jard. Bot. Rio de Janeiro 14: 13. 1956. Type: BRAZIL. SANTA CATARINA: Ilha de Santa Catarina, Sertão da Lagoa, 6.IV.1951, fl., J.A. Rohr 2126 (holotype HB!).

Anathallis laciniata (Barb.Rodr.) F.Barros \& Barberena, comb. nov. Pleurothallis laciniata Barb.Rodr., Gen. Spec. Orchid. 1: 14. 1877. Lepanthes laciniata (Barb.Rodr.) Barb.Rodr., Gen. Spec. Orchid. 2: 68. 1881. Specklinia laciniata (Barb.Rodr.) Luer, Monogr. Syst. Bot. Missouri Bot. Gard. 95: 261. 2004. Panmorphia laciniata (Barb.Rodr.) Luer, Monogr. Syst. Bot. Missouri Bot. Gard. 105: 177. 2006. Type: BRAZIL. MINAS GERAIS: Poços de Caldas, I.1877, J.B. Rodrigues s.n. (holotype RB, destroyed); lectotype, here designated, fig. A, tab. 133, vol. 3 (J. Barbosa Rodrigues, Iconographie des Orchidées du Brésil, reproduced in Sprunger (1996, p. 191)).

Anathallis lobiserrata (Barb.Rodr.) F.Barros \& Barberena, comb. nov. Lepanthes lobiserrata Barb.Rodr., Gen. Spec. Orchid. 2: 63. 1881. 
Pleurothallis lobiserrata (Barb.Rodr.) Cogn. in Martius, Eichler \& Urban, Fl. bras. 3(4): 438. 1896. Specklinia lobiserrata (Barb. Rodr.) Luer, Monogr. Syst. Bot. Missouri Bot. Gard. 95: 261. 2004. Panmorphia lobiserrata (Barb.Rodr.) Luer, Monogr. Syst. Bot. Missouri Bot. Gard. 105: 177. 2006. Type: BRAZIL. RIO DE JANEIRO: Rodeio, I, J.B. Rodrigues s.n. (holotype RB, destroyed); lectotype, here designated, fig. F, tab. 298, vol. 6 (J. Barbosa Rodrigues, Iconographie des Orchidées du Brésil, reproduced in Sprunger (1996, p.426)).

Anathallis marginata (Barb.Rodr.) F.Barros \& Barberena, comb. nov. Lepanthes marginata Barb.Rodr., Gen. Spec. Orchid. 2: 68. 1881. Type: BRAZIL. RIO DE JANEIRO: Rio de Janeiro, Pascoinha, X, J.B. Rodrigues s.n. (holotype RB, destroyed); lectotype, here designated, fig. C, tab. 133, vol. 3 (J. Barbosa Rodrigues, Iconographie des Orchidées du Brésil, reproduced in Sprunger (1996, p.191)). Pleurothallis limbata Cogn. in Martius, Eichler \& Urban, Fl. bras. 3(4): 485. 1896, non Lindl. (1838), nec Cogn. (1896). Specklinia limbata (Cogn.) Luer, Monogr. Syst. Bot. Missouri Bot. Gard. 95: 261. 2004. Panmorphia limbata (Cogn.) Luer, Monogr. Syst. Bot. Missouri Bot. Gard. 105: 177. 2006.

Anathallis muscoidea (Lindl.) F.Barros \& Barberena, comb. nov. Pleurothallis muscoidea Lindl., Edwards's Bot. Reg. 24: 89. 1838. Humboldtia muscoidea (Lindl.) Kuntze, Revis. Gen. Pl. 2: 668. 1891. Specklinia muscoidea (Lindl.) Luer Monogr. Syst. Bot. Missouri Bot. Gard. 112: 121. 2007. Type: Sine loco: C. Loddiges s.n. (holotype K; photo of the holotype SP!).

Anathallis nanifolia (Foldats) F.Barros \& Barberena, comb. nov. Pleurothallis nanifolia Foldats, Bol. Soc. Venez. Ci. Nat. 22(100): 258, fig. 3. 1961. Specklinia nanifolia (Foldats) Luer, Monogr. Syst. Bot. Missouri Bot. Gard. 95: 262. 2004. Panmorphia nanifolia (Foldats) Luer, Monogr. Syst. Bot. Missouri Bot. Gard. 105: 169. 2006. Type: VENEZUELA. BOLÍVAR: por arriba de Raudal Cotua, al sur de La Paragua, 1.VIII.1960, fl., J.A. Steyermark 86725 (isotype NY; photo of the isotype NY!).

Additional material examined: BRAZIL. MATO GROSSO: Chapada dos Guimarães, Cachoeira Véu da Noiva, fl. cult., VI.1983, D.M. Vital s.n. (SP 237321).

Anathallis petropolitana (Hoehne) F.Barros \& Barberena, comb. nov. Pleurothallis petropolitana Hoehne, Arch. Inst. Biol. Defesa Agric. 3: 296, tab.
39, fig. 1. 1930. Specklinia petropolitana (Hoehne) Luer, Monogr. Syst. Bot. Missouri Bot. Gard. 95: 263. 2004. Panmorphia petropolitana (Hoehne) Luer, Monogr. Syst. Bot. Missouri Bot. Gard. 105: 177. 2006. Type: BRAZIL. RIO DE JANEIRO: Petrópolis, I.1928, fl., C. Spannagel 123 (isotype SP!).

Anathallis recurvipetala (Barb.Rodr.) F.Barros \& Barberena, comb. nov. Lepanthes recurvipetala Barb.Rodr., Gen. Spec. Orchid. 2: 62. 1881. Pleurothallis recurvipetala (Barb.Rodr.) Cogn. in Martius, Eichler \& Urban, Fl. bras. 3(4): 439. 1896. Panmorphia recurvipetala (Barb.Rodr.) Luer, Monogr. Syst. Bot. Missouri Bot. Gard. 105: 177. 2006. Type: BRAZIL. RIO DE JANEIRO: Rodeio, pr. Serra do Mar, II, J.B. Rodrigues s.n. (holotype RB, destroyed); lectotype, here designated, fig. C, tab. 298, vol. 6 (J. Barbosa Rodrigues, Iconographie des Orchidées du Brésil, reproduced in Sprunger (1996, p.426)).

Anathallis rubrolimbata (Hoehne) F.Barros \& Barberena, comb. nov. Pleurothallis rubrolimbata Hoehne, Arq. Bot. Estado São Paulo, n.s., f.m., 2: 22, tab. 8, fig. 3. 1946. Specklinia rubrolimbata (Hoehne) Luer, Monogr. Syst. Bot. Missouri Bot. Gard. 95: 263. 2004. Panmorphia rubrolimbata (Hoehne) Luer, Monogr. Syst. Bot. Missouri Bot. Gard. 105: 177. 2006. Type: BRAZIL. RIO DE JANEIRO: Petrópolis, VI.1934, fl., C. Spannagel 379 (holotype SP!).

Anathallis taracuana (Schltr.) F.Barros \& Barberena, comb. nov. Pleurothallis taracuana Schltr., Beih. Bot. Centralbl. 42(2): 91. 1925. Type: BRAZIL. AMAZONAS: Taracuá, Alto Rio Negro, II, G. Hübner 167 (holotype B, destroyed).

Up to now this species is known only from the type collection which was destroyed during the World War II.

Anathallis tigridens (Loefgr.) F.Barros \& Barberena, comb. nov. Pleurothallis tigridens Loefgr., Arch. Jard. Bot. Rio de Janeiro 2: 56, tab. 23. 1918. Specklinia tigridens (Loefgr.) Luer, Monogr. Syst. Bot. Missouri Bot. Gard. 95: 264. 2004 Panmorphia tigridens (Loefgr.) Luer, Monogr. Syst. Bot. Missouri Bot. Gard. 105: 177. 2006. Type: BRAZIL. RIO DE JANEIRO: Rio de Janeiro, Corcovado, 1916, fl., $P$. Occhioni s.n. (holotype RB 8204!).

Additional material examined: BRAZIL. RIO DE JANEIRO: Itatiaia, pr. Rio Campo Belo, IV.1955, fl., A.C. Brade s.n. (HB 2670). 
Anathallis vitorinoi (Luer \& Toscano) F.Barros \& Barberena, comb. nov. Pleurothallis vitorinoi Luer \& Toscano, Selbyana 23: 195, fig. 10. 2002. Specklinia vitorinoi (Luer \& Toscano) Luer, Monogr. Syst. Bot. Missouri Bot. Gard. 95: 264. 2004. Panmorphia vitorinoi (Luer \& Toscano) Luer, Monogr. Syst. Bot. Missouri Bot. Gard. 105: 177. 2006. Type: BRAZIL. SÃO PAULO: São Bernardo do Campo, 19.I.1997, V.P. Castro \& M.A. Campacci s.n. (holotype HUEFS, not found).

This species is generally identified as Anathallis fastigiata. We are unable to find the holotype at the herbarium of the State University of Feira de Santana (HUEFS), and apparently the material has never been deposited there. Although, the description and the illustration allow us to conclude that the species belongs to the genus Anathallis.

The following species, previously known as Pleurothallis mentigera Kraenzl., has the characteristics of the genus Specklinia, and need to be transferred from Pleurothallis sensu stricto. It has a ramicaul shorter than the leaves, with an annulus, the sepals and petals are membranaceous, the lateral sepals are connate, the petals are shorter than the sepals, the lip is hinged, and the column is elongate with a single and ventral stigmatic cavity and ventral anther.

Specklinia mentigera (Kraenzl.) F.Barros \& Barberena, comb. nov. Pleurothallis mentigera Kraenzl., Kongl. Svenska Vetensk. Acad. Handl., n.s., 46(10): 50. 1911. Type: BRAZIL. PARANÁ: Roça Nova, Banhado, XII.1908, fl., P.K. Dusén 7433 (holotype B; photo of the type AMES!).

Pleurothallis magnicalcarata Loefgr., Arch. Jard. Bot. Rio de Janeiro 2: 51, fig. 18B. 1918. Type: BRAZIL. SÃO PAULO: Guaratinguetá, 1916, P.C. Porto s.n. (holotype RB 8285!).

Additional material examined: BRAZIL. MINAS GERAIS: Itamonte, Parque Nacional do Itatiaia, 25.I.2009, fl., F.F.V.A. Barberena 131 (RB).

The following eight binomials, although previously transferred to Anathallis in the papers of Azevedo \& Van den Berg (2005) and Barros (2002, 2003, 2006), have been transferred again by Luer (2007), establishing some illegitimate names for the genus. The same occurred even with a species transferred to Specklinia by Lindley (1830-1840).
Anathallis bleyensis (Pabst) F.Barros, Hoehnea 30(3): 187. 2003. Pleurothallis bleyense Pabst, An. XIV Congr. Soc. Bot. Bras. p. 13, tab. 2, fig. B. 1964. Anathallis bleyensis (Pabst) Luer, Monogr. Syst. Bot. Missouri Bot. Gard. 112: 118. 2007, nom. illeg. Type: BRAZIL. PARANÁ: Lapa, Engenheiro Bley, fl. cult., 20.III.1953, fl., G. Hatschbach 3000 (holotype HB!).

Anathallis graveolens (Pabst) F.Barros, Bradea 11(1): 30. 2006. Pleurothallis graveolens Pabst, Bradea 1(50): 488, fig. B. 1975. Specklinia graveolens (Pabst) Luer, Monogr. Syst. Bot. Missouri Bot. Gard. 95: 260. 2004. Anathallis graveolens (Pabst) Luer, Monogr. Syst. Bot. Missouri Bot. Gard. 112: 118. 2007, nom. illeg. Type: BRAZIL. ESPÍRITO SANTO: Domingos Martins, 24.XI.1970, fl., R. Kautsky 306 (holotype HB!).

Anathallis guarujaensis (Hoehne) F.Barros, Hoehnea 30(3): 187. 2003. Pleurothallis guarujaensis Hoehne, Arq. Bot. Estado São Paulo 1: 15, tab. 5, fig. 1. 1938. Anathallis guarujaensis (Hoehne) Luer, Monogr. Syst. Bot. Missouri Bot. Gard. 112: 118. 2007, nom. illeg. Type: BRAZIL. SÃO PAULO: Guarujá, Ilha de Santo Amaro, praia das Tartarugas, 23.III.1938, fl., F.C. Hoehne s.n. (holotype SP 39228!).

Anathallis imbricata (Barb.Rodr.) F.Barros \& F.Pinheiro, Bradea 8(48): 329. 2002. Pleurothallis imbricata Barb.Rodr., Gen. Sp. Orch. 1: 15. 1877. Anathallis imbricata (Barb.Rodr.) Luer, Monogr. Syst. Bot. Missouri Bot. Gard. 112: 118. 2007, nom. illeg. Type: BRAZIL.J.B. Rodrigues s.n. (holotype RB, destroyed); lectotype: tab. 133, vol. 3 (J. Barbosa Rodrigues, Iconographie des Orchideés du Brésil, designated by Barros \& Pinheiro (2002)). Lepanthes imbricata (Barb.Rodr.) Barb.Rodr., Gen. Sp. Orch. 2: 56. 1882.

Anathallis microphyta (Barb.Rodr.) C.O. Azevedo \& Van den Berg, Kew Bull. 60(1): 137. 2005. Lepanthes microphyta Barb.Rodr., Gen. Sp. Orchid. 2: 67. 1882. Anathallis microphyta (Barb.Rodr.) Luer, Monogr. Syst. Bot. Missouri Bot. Gard. 112: 118. 2007, nom. illeg. Type: BRAZIL. RIO DE JANEIRO: Rodeio, II, J.B. Rodrigues s.n. (holotype RB, destroyed); lectotype: fig. B, tab. 298, vol. 3 (J. Barbosa Rodrigues, Iconographie des Orchideés du Brésil, designated by Azevedo \& Van den Berg (2005)). 
Anathallis reedii (Luer)F.Barros, Bradea 11(1):30. 2006. Pleurothallis reedii Luer, Selbyana 23(2): 186, fig. 5. 2002. Specklinia reedii (Luer) Luer, Monogr. Syst. Bot. Missouri Bot. Gard. 95: 263. 2004. Anathallis reedii (Luer) Luer, Monogr. Syst. Bot. Missouri Bot. Gard. 112: 118.2007, nom. illeg. Type:BRAZIL.BAHIA: 1880, fl., W. Reed et Saunders s.n. (holotype AMES n.v.).

Anathallis sertularioides (Sw.) Pridgeon \& M.W.Chase, Lindleyana 16: 250. 2001. Epidendrum sertularioides Sw., Prodr. p. 122. 1788. Dendrobium sertularioides (Sw.) Sw., Nova Acta Regiae Soc. Sci. Upsal. 6: 83. 1799. Pleurothallis sertularioides (Sw.) Spreng., Syst. Veg. 3: 731. 1826. Specklinia sertularioides (Sw.) Lindl., Gen. Sp. Orchid. Pl., p. 8. 1830. Humboldtia sertularioides (Sw.) Kuntze, Revis. Gen. Pl. 2: 668. 1891. Specklinia sertularioides (Sw.) Luer, Monogr. Syst. Bot. Missouri Bot. Gard. 95: 263. 2003, nom. illeg. Panmorphia sertularioides (Sw.) Luer, Monogr. Syst. Bot. Missouri Bot. Gard. 105: 174. 2006. Type: JAMAICA. Sine loco: fl., O. Swartz s.n. (holotype S!; photo of the holotype SP!).

Pleurothallis tenuissima Rchb.f., Linnaea 18: 399. 1845. Humboldtia tenuissima (Rchb.f.) Kuntze, Revis. Gen. Pl. 2: 668. 1891. Type: MEXICO. Sine loco: F.E. Liebold 620 (holotype W; drawing of the type AMES!).

Pleurothallis trichopoda A.Rich. \& Galeotti, Ann. Sci. Nat., Bot., III, 3: 17. 1845. Humboldtia trichopoda (A.Rich. \& Galeotti) Kuntze, Revis. Gen. Pl. 2: 668. 1891. Type: CUBA. Sine loco: 1856, fl., C. Wright 659 (holotype K; photo of the type AMES!).

Anathallis subnulla (Luer \& Toscano) F.Barros, Bradea 11(1): 31.2006. Pleurothallis subnulla Luer \& Toscano, Selbyana 23(2): 190-194, fig. 8. 2002. Specklinia subnulla (Luer \& Toscano) Luer, Monogr. Syst. Bot. Missouri Bot. Gard. 95: 264. 2004. Anathallis subnulla (Luer \& Toscano) Luer, Monogr. Syst. Bot. Missouri Bot. Gard. 112: 118. 2007, nom. illeg. Type: BRAZIL. BAHIA: Instituto de Botânica de São Paulo sob n ${ }^{\circ}$ 13515, 23.V.1986, fl., A.L.V. Toscano-de-Brito 2293 (holotype HUEFS).

\section{References}

Azevedo, C.O. \& Van den Berg, C. 2005. A new combination in the genus Anathallis(Orchidaceae), and a new record for Bahia State, Brazil. Kew Bulletin 60: 137-138.

Barros, F. 2002. Notas nomenclaturais em Pleurothallidinae (Orchidaceae), principalmente brasileiras. Bradea 8: 293-297.

Barros, F. 2003. Notas taxonômicas sobre espécies brasileiras dos gêneros Catasetum, Isabelia, Veyretia, Acianthera e Anathallis (Orchidaceae). Hoehnea 30: 181-191.

Barros, F. 2004. Taxonomic and nomenclatural notes on Brazilian Orchidaceae. In: Manilal, K.S. \& Sathish Kumar, C. (eds.). Orchid memories: a tribute to Gunnar Seidenfaden. Mentor Books, Calicut. Pp. 7-22.

Barros, F. 2005. Notas taxonômicas para espécies brasileiras dos gêneros Acianthera, Anathallis, Specklinia e Heterotaxis (Orchidaceae). Hoehnea 32: 421-428.

Barros, F. 2006. Seis novas combinações para orquídeas brasileiras. Bradea 11:29-32.

Barros, F. \& Pinheiro, F. 2002. Duas novas combinações em Pleurothallidinae (Orchidaceae) de Grão Mogol (Minas Gerais, Brasil). Bradea 8: 329-330.

Holmgren, P.K.; Holmgren, N.H. \& Barnett, L.C. 1990. Index Herbariorum: the herbaria of the world. $8^{\text {th }} \mathrm{ed}$. New York Botanical Garden, New York. 693p.

Lindley, J. 1830-1840. The genera and species of orchidaceous plants. Ridgways, London. 1963 reprint. A. Asher \& Co., Amsterdam. 553p.

Luer, C.A. 2004. New genera and combinations in Pleurothallidinae. Icones Pleurothallidinarum XXVI. Monographs in Systematic Botany from the Missouri Botanical Garden 95: 253-265.

Luer, C.A. 2006. Icones Pleurothallidinarum XXVIII. Monographs in Systematic Botany from the Missouri Botanical Garden 105: 1-274.

Luer, C.A. 2007. Icones Pleurothallidinarum XXIX. Monographs in Systematic Botany from the Missouri Botanical Garden 112: 1-130.

Luer, C.A. 2008. New combinations in the Pleurothallidinae (Orchidaceae) from the Southern Cone Region of South America. Novon 18: 78-79.

Luer, C.A. \& Toscano-de-Brito, A.L.V. 2002. Miscellaneous new species of Pleurothallidinae from Brazil. Selbyana 23: 181-195.

Pridgeon, A.M. \& Chase, M.W. 2001. A phylogenetic reclassification of the Pleurothallidinae (Orchidaceae). Lindleyana 16: 235-271.

Sprunger, S. (ed.). 1996. João Barbosa Rodrigues Iconographie des orchidées du Brésil, v. 1: The illustrations. Friedrich Reinhardt Verlag, Basle. 540p. 